\title{
(9)
}

\section{GESTIÓN DE RECURSOS PÚBLICOS Y EVALUACIÓN: UN BINOMIO INSEPARABLE}

Políticas públicas bien diseñadas y convenientemente evaluadas mejoran el bienestar no solo económico, sino también la calidad institucional y democrática. El despliegue de abundantes recursos públicos en un corto espacio temporal para hacer frente a la pandemia otorga a la evaluación de las políticas públicas máxima actualidad. En este artículo se repasan experiencias internacionales destacadas en el ámbito de la evaluación de políticas públicas, se profundiza en el caso particular de las revisiones de gasto que realiza la AIReF y se apuntan los retos de evaluación asociados al Plan Nacional de Recuperación y Resiliencia.

Palabras clave: evaluación, políticas públicas, spending review, eficiencia del gasto público, supervisión fiscal, acceso a datos.

Clasificación JEL: H43, H83.

\section{Introducción}

La evaluación de las políticas públicas es un tema recurrente, pero que ha recobrado especial actualidad. Ello no debe sorprender, puesto que hay consenso generalizado, entre academia y gestores, en que políticas públicas bien diseñadas y convenientemente evaluadas mejoran el bienestar no solo económico, sino también la calidad institucional y democrática. Y porque, sin embargo, la práctica se aleja, en muchos aspectos, de lo que recomienda la teoría.

Ahora bien, diría que estamos atravesando un momento que puede permitir dar un salto cualitativo en esta materia. Primero, por

*Presidenta de la AIReF (Autoridad Independiente de Responsabilidad Fiscal).

Versión de abril de 2021.

DOI: https:/doi.org/10.32796/bice.2021.3135.7185 necesidad y oportunidad, ya que los recursos que se están movilizando para dar respuesta a la crisis desencadenada por la pandemia no relajan, en absoluto, la necesidad de optimizar su uso. $Y$ segundo, porque se han realizado progresos importantes en el diseño de bases de datos y en la elaboración de indicadores que permiten proveer de inputs rigurosos y objetivos a los gestores y responsables de las políticas y recursos públicos. Así, por ejemplo, la existencia de bases de microdatos, la mejora de los registros administrativos y plataformas de bases de datos que permiten una más fácil interrelación, junto con las nuevas posibilidades tecnológicas para el tratamiento masivo de datos, abren vías de análisis que no existían hace unos años

En el ámbito internacional hay iniciativas y buenas prácticas que son, desde luego, un $D$ 
referente a tener en cuenta. Cabe destacar los casos del Reino Unido y Estados Unidos, donde ciertamente la evaluación cuenta con tradición y arraigo, y que en los últimos años están dando pasos sustantivos para su impulso y sistematización en una doble dirección: 1) integración plena en el ciclo presupuestario y 2) diseño, desarrollo e implementación de políticas públicas.

En el caso particular del Reino Unido, la evaluación está integrada totalmente con carácter sistemático en el proceso presupuestario. Desde la introducción en 1997 de programaciones presupuestarias a tres años, se vienen realizando evaluaciones ordinarias del gasto (spending reviews) con antelación a cada nuevo periodo plurianual. Estas revisiones se complementan con los denominados comprehensive spending reviews, que tienen mayor alcance temporal y una visión más estratégica, y con los public service agreements, que son acuerdos con departamentos en el que se fijan metas de productos y resultados. Las últimas revisiones de gasto se realizaron a finales del año pasado.

Además, para impulsar el papel de la evaluación en el proceso de gestión de políticas públicas, en 2013 el Gobierno lanzó la iniciativa What Works Network, con la que se promueve el uso de la evidencia para el diseño y prestación de los servicios públicos. Se trata de una red de centros independientes y asociados en diversas áreas que se comprometen a impulsar la oferta y la demanda de evidencia en sus respectivos ámbitos y tratar de adaptar sus outputs a las necesidades del decisor. Para ello, recolectan y sintetizan los datos existentes sobre la eficacia de las políticas públicas y la evalúan contra una serie de indicadores acordados. Además, tratan de hacer frente a los vacíos de evidencia comisionando análisis y apoyan a la Administración en el uso de esta evidencia. Se financian con recursos públicos y privados. Integran centros en diversos ámbitos como sanidad, educación o envejecimiento.

En Estados Unidos, en 2010, se reformó la Government Performance and Results Act (GPRAMA), que obliga a las agencias y departamentos federales a desarrollar, sobre la base de indicadores y metas cuantificables basadas en datos, planes estratégicos y memorias anuales de desempeño, cuya información ha de justificar las solicitudes de asignación presupuestaria. Además de reforzar las bases y requisitos legales de planes y memorias, se crearon nuevos puestos en cada agencia y departamento, entre otros un director de operaciones y un director de desempeño para asegurar un mayor nivel de implementación.

En 2018 se aprobó, por unanimidad, la Foundations for Evidence-Based Policymaking Act, que implementaba las recomendaciones de la Commission on Evidence-Based Policymaking, creada durante la Administración Obama. Con ello se establecía un marco ambicioso de evaluación, amplio y unificado para las diferentes agencias y departamentos federales. Comprende las evaluaciones ex ante y ex post; obliga a las agencias a desarrollar políticas basadas en evidencia y planes de evaluación; así como a fijar una ruta, dentro de su plan estratégico, para identificar y elaborar indicadores pertinentes de programas y políticas, definiendo estrategias, métodos y bases de datos a desarrollar adicionales a las exigidas por GPRAMA. Por otra parte, y para asegurar su puesta en práctica, se encargó a la Office of Management Budget la supervisión de su implementación durante sus cuatro fases (20192022), labor que lleva a cabo mediante la emisión de guías de actuación y metodológicas, identificando áreas prioritarias sobre las $\triangleright$ 
que obtener evidencia, y proponiendo soluciones a los obstáculos de implementación detectados. Adicionalmente, esta ley dotó a los departamentos y agencias de recursos humanos adicionales, con especial atención a la información. La nueva Administración Biden ya ha mostrado su determinación para consolidar esta práctica.

En el ámbito de la Unión Europea, por su innovación al adentrase incluso en el terreno de la economía experimental, cabe destacar a Finlandia. La evaluación, en su concepto amplio, está presente en este país desde los años sesenta, cuando se comenzó a implantar el desarrollo y la utilización de evidencia para hacer reformas del estado de bienestar. Se comenzó principalmente con evaluaciones ex ante. En los años ochenta y noventa, bajo el influjo de las prácticas anglosajonas, se produjeron importantes reformas y se comenzaron a abordar evaluaciones ex post de eficiencia y eficacia, con el establecimiento de grupos de evaluación y el desarrollo de guías de evaluación para las agencias gubernamentales. Así, en 2010 la evaluación en los diferentes ministerios era ya una práctica asentada y, tras nuevas reformas en 2014, cada año el Gobierno adopta un plan de análisis, asesoramiento e investigación que permite reforzar las políticas públicas con evidencia, estableciendo áreas prioritarias de evaluación con un seguimiento de los objetivos. Su actuación se complementa, entre otras, con evaluaciones externas que financia el Consejo de Investigación Estratégica y con la actividad del Consejo de Impacto Regulatorio, de naturaleza independiente, que tiene la misión de realizar evaluaciones ex ante de todas las propuestas legislativas en cartera. No obstante, hay todavía limitaciones en la implementación práctica que la Oficina Nacional de Auditoría ha puesto de relieve.
El compromiso con la evaluación se pone de manifiesto con el experimento que se realizó sobre el ingreso mínimo vital (IMV) durante los años 2017 y 2018 como apoyo a la remodelación de una parte del sistema de seguridad social. En concreto, su objetivo era analizar el impacto en la búsqueda de empleo de la puesta en marcha a nivel nacional de un IMV. La dirección del proyecto se atribuyó a Kela (Instituto de Seguridad Social finlandés) y el experimento se basó en un muestreo aleatorio (175.000 personas) a nivel nacional de personas que estaban recibiendo la prestación básica de desempleo. Se utilizaron técnicas propias de la evaluación, por lo que se creó un grupo de tratamiento de 2.000 desempleados que pasaron a recibir el IMV de cuantía neta similar a la prestación por desempleo, pero no condicionado a la búsqueda de empleo, y se analizó su comportamiento frente al de un grupo de control que siguió recibiendo la prestación por desempleo. Los primeros resultados se presentaron el año pasado, generando gran atención en academia, decisores y medios.

Las instituciones comunitarias están tratando de impulsar estas prácticas, si bien con una visión un poco más modesta y muy centrada, hasta la fecha, en la dimensión presupuestaria. Se puede citar como punto reciente de arranque la declaración del Eurogrupo de septiembre de 2016, en la que se recomendaba a los países del euro utilizar los spending reviews o revisiones de gasto como herramienta para mejorar la calidad de las finanzas públicas. El Eurogrupo acordó una serie de principios que deben orientar dichas revisiones e inició un intercambio regular sobre la práctica y la experiencia de los países.

Esta corriente europea ha llevado al propio Consejo a formular recomendaciones expresas a algunos países para que realicen $D$ 
dichas revisiones. España, así como Bélgica, Francia, Portugal y Malta, recibieron en 2017 una recomendación al respecto. En concreto, a España se le recomendó realizar una revisión integral del gasto público. Más recientemente, con la crisis de la pandemia ya extendida, el pasado otoño se recomendaba a todos los países del euro prestar atención a la calidad de las medidas presupuestarias, así como hacer uso de las revisiones de gasto para focalizarlo en las necesidades de la recuperación y la resiliencia.

Sea por impulso comunitario o por iniciativa propia, España está dando pasos importantes que evidencian un grado de compromiso que destaca entre los países del euro. Tanto a nivel de la Administración central como de las comunidades autónomas, se han adoptado iniciativas para revisar políticas de gran relevancia económica y presupuestaria como la sanidad, la educación, las políticas activas de empleo o importantes beneficios fiscales. Se percibe, además, el compromiso de que se convierta en un ejercicio continuado en el tiempo y no sea una práctica puntual derivada de una moda o presión externa. Esta motivación política es una premisa esencial, y el hecho de que se esté abordando por varios niveles de la Administración al mismo tiempo constituye un buen punto de partida.

Es en este contexto en el que se debe entender la incursión de la AIReF en el ámbito de la evaluación en profundidad de políticas sectoriales basada en la evidencia. En 2017 el Gobierno encargó a la AIReF la revisión integral del gasto público solicitada por las instituciones comunitarias. Se trata de un ejercicio de tres años de duración del que ya se han ejecutado las dos primeras etapas y se está en fase de concretar la tercera. Incluso con anterioridad, varias comunidades autónomas se dirigieron a la AIReF para realizar revisiones de políticas en el ámbito de sus competencias y se siguen incrementando las solicitudes procedentes de los Gobiernos autonómicos. Como resultado, hasta la fecha, la AIReF ha analizado y publicado los resultados de catorce políticas y están en curso otras ocho a las que se sumarán las correspondientes a la tercera fase del spending review de la Administración central.

\section{La AIReF en el ámbito de la evaluación: retos y decisiones estratégicas}

Para la AIReF, la evaluación ha supuesto un doble reto. Un reto organizativo, puesto que la AIReF no estaba dimensionada para estas nuevas tareas. Se carecía de los recursos humanos, de infraestructura de gestión de los datos e información que requiere este tipo de análisis, así como de experiencia de dirección en materia de evaluación. En segundo lugar, y ligado a lo anterior, un reto reputacional en caso de no ser capaces de igualar los estándares que han caracterizado nuestros análisis en el ámbito macroeconómico y presupuestario.

Además, la AIReF no ha sido ajena a los retos comunes que afrontan las instituciones españolas que realizan evaluaciones. Entre ellos, cabe destacar las dificultades para entablar una colaboración constructiva con los gestores de las políticas evaluadas. Falta mucho para que la evaluación sea percibida como un instrumento útil para el gestor. La cultura de rendición de cuentas y análisis por resultados no está tan extendida en España como en los países antes citados. El sesgo hacia evaluaciones de tipo más fiscalizador o procedimental, y con escasa integración en el proceso presupuestario, puede estar detrás de $\square$ 
estas reticencias. Asimismo, la AIReF ha tenido que incorporar a su acervo metodologías nuevas que requieren una especialización todavía poco extendida o muy limitada hasta la fecha al mundo netamente académico. Como otras instituciones, también se han debido resolver los problemas de acceso a la información, como me referiré posteriormente. $\mathrm{Y}$ a todo ello se ha de sumar la propia naturaleza descentralizada de la Administración pública, que complica aún más la evaluación de políticas de carácter transversal.

Si bien aún seguimos haciendo frente a estos retos propios y comunes, y somos conscientes de nuestras limitaciones y ámbitos de mejora, creo que hemos sido capaces de dar una respuesta satisfactoria, como evidencian estos tres primeros años experiencia. Ello ha sido resultado de la combinación de dos factores: 1) algunas características intrínsecas a la configuración institucional de la AIReF y 2) las decisiones que hemos ido adoptando para responder a los retos citados.

El elemento diferenciador fundamental de la AIReF es la sinergia entre sus funciones de supervisión fiscal y la evaluación. La revisión en profundidad de ingresos y gastos está intrínsecamente relacionada con la supervisión fiscal y constituye una salvaguarda de la sostenibilidad financiera, que es el mandato último atribuido a la AIReF. De hecho, en el desarrollo de esta función de supervisión, ya habíamos incidido en la necesidad de evaluar la eficiencia y eficacia del gasto como parte integral de la sostenibilidad de las finanzas públicas. Es más, para poder realizar nuestras propias previsiones fiscales, nos habíamos adentrado en el análisis en profundidad de políticas sectoriales de mayor relevancia cuantitativa, habíamos empezado a construir bases de datos e indicadores de eficiencia como los del ámbito local y, por supuesto, algunas de nuestras herramientas de análisis permiten atender esa doble vertiente. Por poner algún ejemplo, los modelos de microsimulación desarrollados sobre figuras impositivas nos permiten atender demandas tanto de previsiones fiscales como de evaluación de dichas figuras para analizar eficacia o efectos redistributivos. En definitiva, más que excluyentes, la supervisión fiscal y la evaluación son complementarias y entran de lleno en el ámbito de la AIReF.

Además, el ámbito de actuación de la AIReF se extiende a todos los niveles de la Administración pública. La naturaleza orgánica de la ley de creación de la AIReF y las provisiones relativas al deber de colaboración facilitan «legalmente» que la AIReF se haya podido adentrar en políticas de gasto que afectan tanto a la Administración central como a las Administraciones territoriales. Cabe señalar, como ejemplo, nuestras evaluaciones del gasto en sanidad o en políticas activas de empleo, con competencias compartidas.

Por exigencias europeas, las instituciones fiscales independientes como la AIReF deben contar con una fuerte base legal para garantizar su independencia y autonomía funcional, que son dos requisitos irrenunciables del evaluador. El legislador ha velado por la independencia de la AIReF e incluso, el ser una institución nueva, le ha permitido introducir prácticas novedosas, como las relativas al nombramiento de la presidencia o requisitos técnicos de la dirección. Pocas instituciones requieren legalmente el respaldo parlamentario que se exige del presidente de la AIReF ni una experiencia tan profunda. Por otra parte, el presidente disfruta de una capacidad de gestión presupuestaria y de recursos humanos que, si bien no iguala plenamente a otras entidades evaluadoras, sí le ha permitido sortear las carencias $\triangle$ 
mencionadas de recursos y estructura, aunque hay camino por recorrer. Además, frente al predominio de instituciones en las que las decisiones se adoptan de forma colegiada, el carácter unipersonal de la AIReF le ofrece agilidad y unidad de criterio. Los posibles rasgos menos positivos asociados a esta configuración presidencialista se compensan con una estricta rendición de cuentas y el contrapeso de su Comité Directivo y el apoyo del Consejo Asesor.

Con este punto de partida, hemos ido afrontando los retos comunes de todo evaluador, con una serie de decisiones estratégicas que nos están permitiendo configurar un estilo de evaluación propio que tiene en cuenta los principios y buenas prácticas en esta materia. En primer lugar, el énfasis en las evaluaciones de resultados. Existen organismos públicos que ya realizan evaluaciones en profundidad de las políticas públicas, y muchas se han adentrado en el ámbito de los resultados, pero todavía con carácter marginal. Sin renunciar a los aspectos estratégicos y procedimentales, en la AIReF predomina el análisis de eficiencia, eficacia y equidad de las políticas públicas. De algún modo, se cubre un hueco en materia de evaluación que complementa los otros tipos de análisis. Por supuesto, no se trata de enfoques excluyentes, ni de parcelar evaluaciones por tipos de entidades, sino todo lo contrario. Considero que todos los organismos evaluadores públicos y privados deben trabajar de la mano para cubrir todos los ámbitos de la evaluación que, en cada caso, requieren unas competencias, especializaciones y metodologías muy distintas.

En segundo lugar, en línea con las mejores prácticas, se ha hecho un esfuerzo por utilizar los datos y microdatos disponibles para cada evaluación. Utilizamos desde datos de consumo de medicamentos (base de datos Alcántara), la base nacional de subvenciones, el sistema integrado de información de universidades, beneficiarios de políticas activas de empleo a los microdatos anonimizados de la Agencia Tributaria o los millones de billetes de avión vendidos en los últimos años, por poner algunos ejemplos. Así mismo, se emplean análisis cualitativos mediante la elaboración de encuestas de captura de información, entrevistas, grupos focales o análisis con expertos. Por supuesto, la riqueza informativa se explota combinando distintas fuentes de información y cruzando bases de datos. Todo ello ha sido posible gracias a la colaboración de los productores de esta información y el compromiso institucional con estas evaluaciones.

Ahora bien, el acceso a la materia prima necesaria para evaluar con base en la evidencia fue y sigue siendo uno de los grandes retos a los que nos enfrentamos todos los evaluadores. España cuenta con una riqueza incalculable de datos administrativos y económicos, pero no están preparados para su explotación. Los datos están dispersos entre distintos organismos sin comunicación entre ellos, problema que se agudiza aún más por la descentralización. En la AIReF, el acceso a la información resultó complejo no solo por la existencia de reticencias iniciales a compartir determinada información, hasta entonces nunca utilizada para la investigación, que generaba además temores sobre su uso y confidencialidad, sino, también, por la falta de una preparación de los datos para llevar a cabo análisis de calidad.

Por ello, a resultas de nuestra experiencia, el pasado 7 de octubre publicamos una opinión sobre el acceso a datos administrativos, abogando por una estrategia que permita un cambio en esta materia. La experiencia de otros países más avanzados nos ha llevado a proponer un modelo que permita sistematizar el $D$ 
acceso de forma fácil y segura, con posibilidad de cruce entre distintas fuentes, con garantías de confidencialidad y uso responsable. Un primer paso en este sentido sería la puesta a disposición de toda la información utilizada en los estudios de la primera y la segunda fase del spending review mediante una base de datos disponible para la investigación. En definitiva, de nada sirve tener una gran riqueza de datos si estos no pueden ser explotados. En un mundo que avanza de manera vertiginosa hacia la economía de la información, la Administración pública, el mayor tenedor de datos de un país, no puede quedarse atrás. Solo los datos nos permitirán identificar de manera sólida y fundamentada a qué debemos destinar unos recursos cada vez más escasos.

En tercer lugar, versatilidad e idoneidad metodológica. El tipo de evaluaciones que hacemos requiere utilizar metodologías adaptadas a la especificidad de cada evaluación. Planificar una evaluación es tan importante como su realización, e invertimos mucho tiempo en ello. Combinamos las técnicas cuantitativas y cualitativas que consideramos se adaptan mejor a cada proyecto. Hemos realizado desde microsimulaciones y análisis de impacto hasta estudios de benchmarking, encuestas o entrevistas. Por ejemplo, en el ámbito de la evaluación de los beneficios fiscales ha predominado el análisis de eficacia y consideraciones redistributivas. Se han utilizado técnicas cuantitativas de análisis de impacto (regresiones en discontinuidad, o diferencias en diferencias, variables instrumentales) y se han realizado análisis de elasticidades y microsimulaciones. En los estudios sobre sanidad, las técnicas cualitativas han sido una parte esencial, cubriendo desde la revisión documental y normativa, entrevistas con expertos, cuestionarios o visitas a los equipos de dirección y gestión de hospitales cubriendo toda la geografía nacional. Y en todas ellas siempre hay un esfuerzo por mirar las mejores prácticas, sea en el ámbito nacional o internacional.

En cuarto lugar, nuestras evaluaciones buscan alcanzar conclusiones con las que identificar propuestas de factible implementación. El fin último de todo este esfuerzo evaluador no es otro que mejorar la asignación de recursos. Así, desde la AIReF todas nuestras evaluaciones concluyen con propuestas claras y que consideramos factibles a distintos horizontes temporales. Por supuesto, no siempre son propuestas cerradas, y, en muchas ocasiones, ofrecemos baterías de opciones al decisor. Pero, en todos los casos, tratamos de ofrecer inputs útiles. Y ello nos obliga a dar un enfoque pragmático que busca un adecuado equilibrio entre investigación y presentación de resultados, ya que los estudios no se pueden dilatar indefinidamente en el tiempo. Pero de nada sirve este pragmatismo en nuestras evaluaciones si no existe también un compromiso para, al menos, considerar la posible implementación de las propuestas. Aunque, obviamente, la implementación es algo que no corresponde a la AIReF, estamos haciendo un seguimiento del grado de implementación, tratando de construir un diálogo con las Administraciones responsables para explicar nuestras conclusiones.

Estos estándares o rasgos distintivos que nos hemos marcado para nuestras evaluaciones nos han llevado a definir un esquema de trabajo abierto que incorpora recursos externos. Ello nos permite, por un lado, superar las limitaciones de recursos humanos de la institución. Pero, también, contar con los mejores expertos en cada una de las materias analizadas. Es prácticamente imposible aglutinar en una sola institución especialistas en metodologías, tratamiento de datos y políticas sectoriales que requieren las evaluaciones de la AIReF. Uno de $\triangleright$ 
los retos de la AIReF es una integración exitosa del factor humano con perfiles y tareas muy distintos. Estos equipos multidisciplinares son un activo, pero también un factor de complejidad en la dirección. Aunque a medida que vamos evaluando vamos adquiriendo el know-how y una capacidad de trabajo más autónoma, el conocimiento y la experiencia de colaboradores científicos siguen siendo pieza clave de nuestras evaluaciones. Sin embargo, la falta de recursos humanos suficientes sigue siendo un reto dada la creciente demanda de estudios. En este sentido, y como ya he manifestado en numerosas ocasiones, considero fundamental que la AIReF cuente con una estructura interna sólida y consolidada que permita hacer de la evaluación otra de las áreas clave de la institución.

Por último, en todo este proceso la comunicación es fundamental. El carácter, a veces, demasiado técnico de las evaluaciones dificulta la comprensión por parte de un público no especializado. Por ello, hemos afrontado este reto común con un esfuerzo divulgativo extra. Utilizamos herramientas sencillas como infografías o vídeos que acerquen nuestros análisis a los ciudadanos. Debemos ser capaces de explicar que la evaluación de políticas públicas es una herramienta clave en la toma de decisiones para optimizar los recursos. No se trata necesariamente de gastar menos, sino de cómo gastar mejor. $Y$ los datos ofrecen un punto de partida para dar respuesta a esta pregunta.

\section{La sostenibilidad fiscal y la disponibilidad de recursos europeos redoblan las necesidades de evaluación}

La evaluación adquiere más relevancia, si cabe, en el actual contexto de crisis en el que nos encontramos, cuyo fuerte impacto en las finanzas públicas va a exigir decisiones para garantizar la sostenibilidad de la deuda pública. Frente a la práctica de reducciones lineales de gasto, las evaluaciones de eficiencia y eficacia permiten identificar qué políticas funcionan o dónde fallan, cómo redirigir el gasto y cómo rediseñar aquello que no está alcanzando los objetivos para los que fue creado. Parece necesario que la evaluación se integre en todas las etapas del proceso presupuestario de todos los niveles de la Administración.

En este sentido, cabe hacer mención a lo señalado por la Comisión Europea (2021), en su comunicación del pasado 3 de marzo, en sus recomendaciones a los países miembros a la hora de diseñar sus políticas fiscales: «All Member States should focus on the composition and the quality of public finances». La crisis sanitaria y económica provocada por la pandemia ha exigido la puesta en marcha de toda una batería de medidas de apoyo a la economía. La activación de la cláusula de escape por parte de la Comisión Europea ha permitido a los países miembros desalinearse de las reglas normalmente exigidas en el Pacto de Estabilidad y Crecimiento, con el fin de que estos puedan dar una respuesta rápida y contundente a la crisis sin preocuparse por el cumplimiento de las reglas fiscales. Sin embargo, no podemos perder de vista el medio plazo y la necesidad de diseñar un plan que devuelva las finanzas públicas a una senda sostenible.

La gravedad de la crisis de la COVID-19 ha generado una respuesta comunitaria sin precedentes, que hará que la consolidación de la cultura de evaluación de las políticas públicas se torne especialmente relevante durante los próximos años. En julio de 2020, los países de la Unión Europea acordaron un ambicioso $\triangleright$ 
paquete de medidas (Next Generation EU) ${ }^{1}$ dotado con 750.000 millones de euros, de los que 672.500 millones se destinarán al denominado Mecanismo de Recuperación y Resiliencia (en adelante, el Mecanismo).

El objetivo del Mecanismo es, por un lado, contribuir a la recuperación de los países de la UE y, por otro, desarrollar economías robustas, sostenibles y resilientes frente a futuras crisis. Los desembolsos, en forma de transferencias y préstamos, están condicionados a la presentación por parte de los Estados miembros de Planes de Recuperación y Resiliencia nacionales. Estos planes tendrán que incluir una descripción pormenorizada de las reformas e inversiones proyectadas en línea con las prioridades marcadas por la $U^{2}$, y los desembolsos periódicos dependerán del cumplimiento de objetivos cuantitativos e hitos cualitativos definidos por cada Estado miembro. A España le corresponden casi 70.000 millones de euros en transferencias y 72.000 en préstamos. Estos fondos —que se deberán absorber en el periodo 2021 2026 - serán recibidos en su totalidad si se cumple con los compromisos definidos en el plan.

Este Mecanismo supone un hito en el proceso de construcción de la Unión Europea. Se trata de un mecanismo de solidaridad temporal que podría abrir la vía hacia la creación de una capacidad de estabilización central permanente en la zona euro, lo que supondría un gran avance en el diseño de una unión económica y monetaria más completa. Por otro lado, la

\footnotetext{
Este paquete se añade a la creación de una nueva línea de apoyo del Mecanismo de Estabilidad (MEDE), el Instrumento Europeo de Apoyo Temporal para Atenuar los Riesgos de Desempleo en una Emergencia (instrumento SURE, por sus siglas en inglés), y el nuevo Marco Financiero Plurianual 2021-2027.

2 Organizadas en torno a seis pilares: transición ecológica, transformación digital, crecimiento inteligente, sostenible e inclusivo, cohesión social y territorial, sanidad y capacidad de respuesta a crisis, y políticas para la próxima generación como educación y formación.
}

necesidad de acompañarlo de un programa de inversiones y reformas estructurales diseñado por cada Estado miembro, que condiciona el desembolso de fondos, supone una oportunidad de aumentar el crecimiento potencial de la economía española a largo plazo y el bienestar social.

El desafío que supone la gravedad de la crisis derivada de la pandemia, la cantidad de fondos que debe absorberse y la condicionalidad de los desembolsos del Mecanismo, unida a la importancia que tiene el buen funcionamiento de este primer mecanismo de solidaridad fiscal europeo, provoca que la evaluación en todas las etapas del proceso adquiera una especial relevancia.

Las especificidades en el diseño del Mecanismo conllevan algunas particularidades en cuanto al tipo de evaluación que es preciso llevar a cabo. Estas peculiaridades pueden resumirse en tres elementos.

El primero de ellos se refiere a la rapidez. Es necesario que el plan se diseñe con rapidez, como se está haciendo, para que los fondos disponibles puedan comenzar a ejecutarse lo antes posible y se evite un daño permanente en los tejidos productivos. Ante el reto sin precedentes de absorción de fondos ${ }^{3}$, se ha flexibilizado el marco jurídico para simplificar los procedimientos de gestión ${ }^{4}$. Esta medida puede resultar necesaria en una economía como la española que necesita aumentar la agilidad en la planificación, asignación y ejecución de proyectos, pero ello no debe representar una reducción de los controles necesarios para lograr una buena $D$

\footnotetext{
3 Los fondos del paquete Next Generation EU se unen a los fondos por absorber del Marco Financiero Plurianual 2014-2020 y del Marco Financiero Plurianual 2021-2027.

4 A través del Real Decreto-ley 36/2020, de 30 de diciembre, por el que se aprueban medidas urgentes para la modernización de la Administración pública y para la ejecución del Plan de Recuperación, Transformación y Resiliencia (BOE de 30 de diciembre de 2020).
} 
asignación de los recursos, en los que la evaluación ex post cobra especial relevancia.

El segundo elemento tiene que ver con el alcance del Plan de Recuperación, Transformación y Resiliencia (en adelante el Plan), tanto en el ámbito temporal como en el material. Por un lado, los planes deben contener iniciativas en el horizonte comprendido entre los años 2020 y 2026; se trata, por tanto, de un amplio periodo de tiempo a lo largo del cual tanto la coyuntura de la economía española como las necesidades de intervención pública irán evolucionando. Esta ventana temporal abre también la posibilidad de que a través de la evaluación se vayan modulando o revisando aquellos aspectos del Plan cuyos resultados no sean satisfactorios.

Por otro lado, en el ámbito material, los Estados miembros tienen que elaborar un programa coherente de reformas e iniciativas de inversión que proporcione una visión global y una prospectiva de las actuaciones que el Estado miembro se compromete a emprender en los próximos años. Este carácter global ha de complementarse con una importante granularidad en lo que se refiere a las medidas específicas. En concreto, según las directrices publicadas por la Comisión Europea, los planes de recuperación y resiliencia deben estar compuestos por componentes muy detallados. $Y$ todo ello dentro de un conjunto muy amplio de ámbitos de política económica relacionados con:

[...] la cohesión económica, social y territorial, la transición ecológica y digital, la salud, la competitividad, la resiliencia, la productividad, la educación y las capacidades, la investigación y la innovación, el crecimiento inteligente, sostenible e inclusivo, el empleo, la inversión y la estabilidad de los sistemas financieros. (artículo 3 del Reglamento (UE)
$\operatorname{COM}(2020) 408$ final del 28 de mayo de 2020, por el que se establece un Mecanismo de Recuperación y Resiliencia)

El tercer elemento está relacionado con el hecho de que el desembolso de los fondos está ligado al desempeño y no a la ejecución de los proyectos, como ocurría con los fondos europeos anteriormente existentes. De este modo, la Comisión autorizará los desembolsos en función del cumplimiento satisfactorio de un grupo de hitos y objetivos que reflejen los avances en las distintas reformas e inversiones del Plan.

En definitiva, la celeridad de la elaboración del Plan, junto con su horizonte temporal y el amplio abanico de elementos que debe contener, hace muy relevante identificar de manera clara los elementos de evaluación que pueden contribuir a maximizar sus efectos positivos y garantizar el uso eficiente y eficaz de la gran cantidad de recursos públicos que se van a ejecutar.

En concreto, las características específicas del Plan de Recuperación y Resiliencia ponen el foco en un triple eje de evaluación para cada componente del mismo:

- La dimensión ex ante, que engloba el diseño de las políticas y la necesidad de seleccionar buenos proyectos que contribuyan a mejorar las perspectivas de un crecimiento sólido, sostenible y equitativo en el futuro.

- La dimensión de ejecución, que ha de ser diligente para que se desplieguen los efectos positivos del fondo cuanto antes, y aprovechar así el estímulo adicional que lleva aparejado el hecho de que la inversión sea coordinada con el resto de los socios de la UE. Al mismo tiempo, ha de garantizarse un control adecuado del uso de los recursos públicos. 
- La dimensión ex post, que abarca la evaluación de los efectos que esas políticas e inversiones vayan generando. Esto es crucial para, dado el horizonte temporal del Plan, poder ir corrigiendo aspectos concretos del diseño de los distintos componentes de manera que se reduzcan posibles ineficiencias y se potencien los efectos positivos.

Por lo tanto, diseñar un sistema adecuado de evaluación del Plan es a la vez crucial y complejo, ya que a partir del año que viene y durante su vigencia se solaparán, en un mismo ejercicio, las necesidades de evaluación ex ante y ex post, además del control de la ejecución de sus distintos componentes.

En este sentido, se echa en falta, ahora más que nunca, la existencia de un Consejo Nacional de Productividad que habría facilitado notablemente el diseño y seguimiento de los proyectos y podría haber contribuido a su evaluación, tal y como sucede en el caso de Países Bajos, donde será esta institución la encargada de la evaluación. Estas instituciones fueron creadas en numerosos países europeos siguiendo una recomendación de la Comisión Europea de 2016 en el marco del proceso de fortalecimiento de la gobernanza europea que tuvo lugar tras la crisis financiera y soberana. Una institución de estas características podría haber ayudado a identificar aquellas áreas en las que la intervención pública puede contribuir en mayor medida al aumento de nuestro potencial de crecimiento a medio plazo.

En las circunstancias actuales, la Intervención General de la Administración del Estado (IGAE) constituye una institución con experiencia para llevar a cabo un control robusto de la ejecución de los fondos en la fase de implementación de cada componente.

Pero es fundamental complementar lo anterior con una evaluación ex post, que permita incorporar las lecciones extraídas de las primeras fases de ejecución de un determinado componente para mejorar la eficiencia, la eficacia o la coherencia de las políticas a lo largo de los años de vigencia del Plan (Esquema 1). La AIReF ha acumulado a lo largo de estos últimos años una sólida experiencia en la evaluación ex post de políticas públicas y está, por tanto, favorablemente situada para llevar a cabo estas tareas en el futuro, contribuyendo así al mejor uso de los fondos, de los que se beneficiará la economía española en los próximos años.

No obstante, dada la amplitud de políticas que cabe esperar que contenga y el grado de innovación de algunas líneas de inversión $\triangleright$

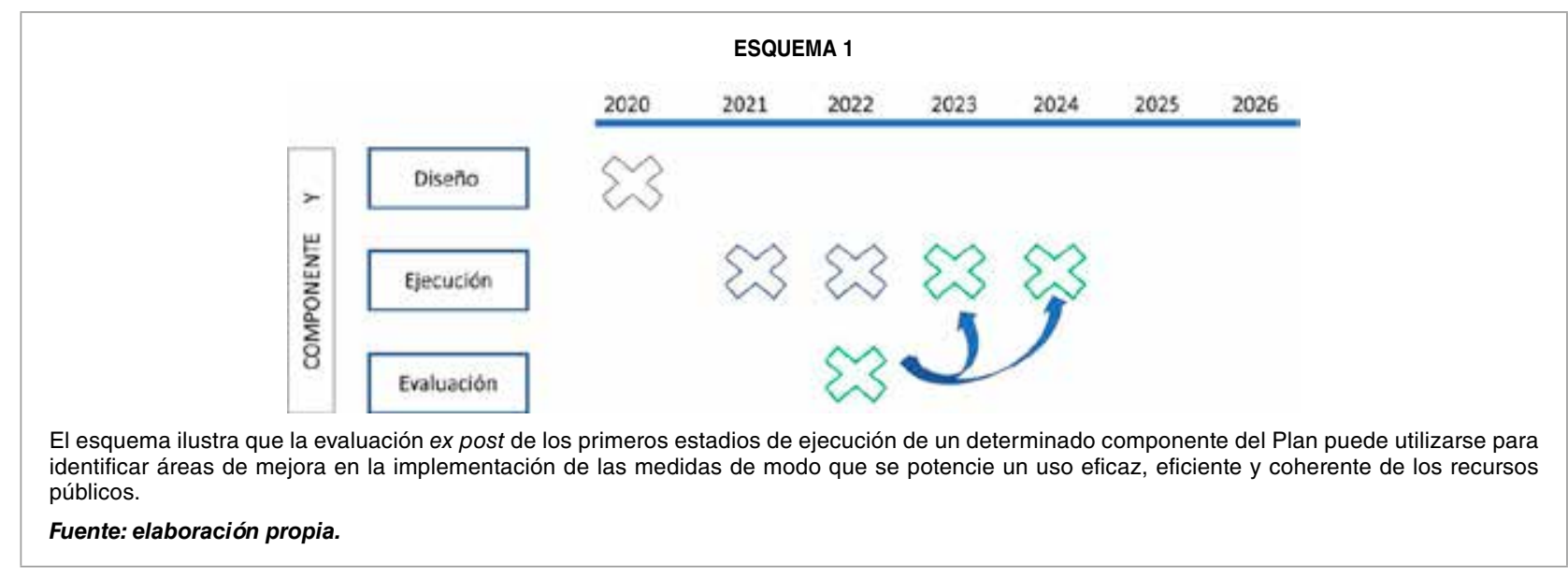


- como la transición ecológica o la digitalización, áreas a las que deben ir destinados al menos el $37 \%$ y el $20 \%$ de los fondos respectivamente-, en esta labor deberían participar varias instituciones independientes con conocimientos técnicos en el área.

\section{Conclusiones}

Por su propia naturaleza de institución supervisora de la salud de las finanzas públicas, la AIReF está progresivamente desarrollando experiencia en materia de evaluación. La revisión en profundidad de las políticas de ingresos y gastos públicos constituye una salvaguarda de la sostenibilidad financiera, que es el mandato último atribuido a la AIReF, y por ello se vienen analizando políticas desde los inicios de nuestra actividad. Pero, sin lugar a duda, la revisión del gasto a tres años, encargada por la Administración central, en línea con las recomendaciones comunitarias, está constituyendo un hito diferenciador. Es más, la intención de mantener esta práctica en el futuro como una actividad regular, son noticias alentadoras.

Ninguna institución puede dar respuesta en solitario a las necesidades de evaluación actuales. Desde la AIReF estamos acumulando experiencia, fundamentalmente, en el ámbito de evaluaciones ex post y centradas en análisis de eficacia, eficiencia y aspectos distributivos, aunque sin descartar por ello otras evaluaciones de tipo más estratégico. Otras instituciones pueden tener ventajas en evaluaciones más de control, ejecución o de diseño. Lo relevante es poder ir definiendo una arquitectura de evaluación que permita su extensión en todos los niveles, y, en este sentido, las prácticas de los países más avanzados pueden ser un referente.
En un mundo tan especializado como el de la evaluación, incluso las iniciativas para compartir e impulsar la generación de la evidencia sobre políticas públicas son atractivas.

El reto de la evaluación requiere, además, resolver los problemas de acceso a la información que toda evaluación rigurosa y basada en la evidencia requiere. La experiencia de la AIReF nos ha llevado a proponer, en línea con los países más avanzados, una estrategia nacional que permita sistematizar de forma fácil, con garantías de confidencialidad y uso responsable, la información disponible en las Administraciones públicas. Y, como paso más inmediato, hemos recomendado la puesta a disposición de toda la información utilizada por la AIReF en las fases ya realizadas de las revisiones de gasto de la Administración central.

Se está atravesando un momento crítico para consolidar en España la evaluación como una actividad regular y extendida en la gestión de las políticas públicas y, en particular, para sustentar las decisiones presupuestarias. Si antes de la pandemia la propia sostenibilidad de las cuentas públicas aconsejaba prestar especial atención a la calidad de las finanzas públicas para optimizar el uso de los recursos públicos, tras ella se ha redoblado esta necesidad. Los abundantes recursos públicos que se están desplegando en todos los niveles de la Administración aconsejan también que el Plan de Recuperación y Resiliencia cuente con su propio esquema de evaluación.

\section{Bibliografía}

Real Decreto-ley 36/2020, de 30 de diciembre, por el que se aprueban medidas urgentes para la modernización de la Administración pública y para la ejecución del Plan de Recuperación, Transformación y Resiliencia. Boletín Oficial $\triangleright$ 
del Estado, n. 341 , de 31 de diciembre de 2020, pp. 126733 a 126793. https://www.boe.es/eli/es/ rdl/2020/12/30/36/dof/spa/pdf

Ley Orgánica 6/2013, de 14 de noviembre, de creación de la Autoridad Independiente de Responsabilidad Fiscal. Boletín Oficial del Estado, n.ำ 274, de 15 de noviembre de 2013, pp. 91298 a 91310. https://www.boe.es/eli/es/lo/2013/11/14/6

Unión Europea (2020). Comunicación (COM (2020) 408 final) de la Comisión, de 28 de mayo de
2020, sobre la Propuesta de Reglamento del Parlamento Europeo y del Consejo por el que se establece un Mecanismo de Recuperación y Resiliencia. Diario Oficial de la Unión Europea. https://ec.europa.eu/transparency/regdoc/ rep/1/2020/ES/COM-2020-408-F1-ES-MAINPART-1.PDF

Unión Europea (2020). Plan de recuperación para Europa: NextGenerationEU. https://ec.europa. eu/info/strategy/recovery-plan-europe_es\#next generationeu 
